\title{
Temperature and Denaturing Gradient Gel Electrophoresis Analysis of 16S rRNA from Human Faecal Samples
}

\author{
Antoon D.L. Akkermans, * Erwin G. Zoetendal, Christine F. Favier, Hans G.H.J. Heilig, \\ Wilma M. AkKermans-van Vliet and Willem M. de Vos \\ Laboratory of Microbiology, Wageningen University, Hesselink van Suchtelenweg 4, 6703 CT Wageningen, The Netherlands \\ Received for publication, December 15, 1999
}

The gastrointestinal tract (GIT) of mammals harbours a complex community of obligate and facultative anaerobic bacteria. The composition of the GIT microbiota is dependent on the physiological condition, age, genetics, and diet of the host. During the past 5 years a number of molecular fingerprinting methods have been developed to characterise complex communities based on 16S rRNA sequence diversity. This paper describes the use of temperature and denaturing gradient gel electrophoresis (T/DGGE) of bacterial 16S rRNA/DNA in faecal samples from humans, in which special attention was given to the quantification of the sequence diversity. After birth the GIT community develops into a relatively simple community consisting of 1-8 major types within three months. Adults show more complex, but remarkably constant patterns. These patterns are hardly affected by changes in diet. Significant differences were observed between different individuals, particularly between genetically unrelated persons. In general, bacterial communities of faecal samples from genetically related adults (i.e. twins, brothers, sisters) show higher similarity of DGGE banding patterns than those from genetically unrelated individuals, although occasionally all persons in one family show highly similar profiles. The DGGE banding patterns of humans are significantly different from those of other mammals, such as pigs, gorillas and cats, indicating that genetic factors of the host affect the composition of the GI-tract flora. The major bacterial groups were identified by cloning and sequencing of the dominant 16S rDNA molecules. At least three sequences with highest homology to Ruminococcus obeum and Eubacterium halii and Fusobacterium prausnitzii were present in all human subjects and are likely to play a universal role in the GI-tract. Other sequences were found in variable ratios in different individuals.

Key words: GI tract; 16S rRNA; DGGE

\section{INTRODUCTION}

The gastrointestinal tract (GIT) harbours a large number of bacteria from different taxonomic groups. The bacterial diversity is affected by the diet as well as the genetic background and physiological state of the host. In the past the composition of the GIT microbial community was investigated by plate counting and other culture-dependent methods $(10,14,24)$. These methods are highly sensitive and accurate to monitor viable micro-organisms from which the growth requirements are known. However, the real world harbours many bacteria from which the growth requirements are still unknown or which are in a dormant state. Such "viable but not cultivable cells" have received much attention, particularly after the initial reports on viable but nonrecoverable pathogens in aquatic systems $(6,23)$. Besides this problem, it remains extremely difficult to obtain a rapid overview of all micro-organisms in an

*Corresponding author. Mailing address: Laboratory of Microbiology, Wageningen University, Hesselink van Suchtelenweg 4, 6703 CT Wageningen, The Netherlands. Phone: +31-317-483486. Fax: +31-317-483829. ecosystem, because there is a large variety of growth requirements among bacteria. Due to these limitations of conventional culture-dependent detection techniques, new DNA-based approaches have been developed on which the presence and identification of the bacteria is simply based on the sequence diversity in the genome (1-4). Special attention has been given to the sequence diversity of ribosomal RNA (rRNA) genes. These genes occur in all living cells with the same function, i.e. involvement in the synthesis of proteins. In bacteria there are three types of rRNA genes that encode molecules of different sizes, i.e. 5S, $16 \mathrm{~S}$ and $23 \mathrm{~S}$ rRNA in which most attention has been given to the 16S rRNA gene resulting in a database containing more than $10,00016 \mathrm{~S}$ rDNA sequences. The most common used database for rRNA sequence analysis is that of the Ribosomal Database Project, RDP $(15,17)$. The characterisation of bacteria in complex ecosystems by $16 \mathrm{~S}$ rDNA sequences diversity has become a common practice, especially by using PCR-based techniques. By using conserved primers, PCR-generated amplicons from all bacteria can be obtained and subsequently separated by denaturing (11) or temperature (22) gradient gel elec- 


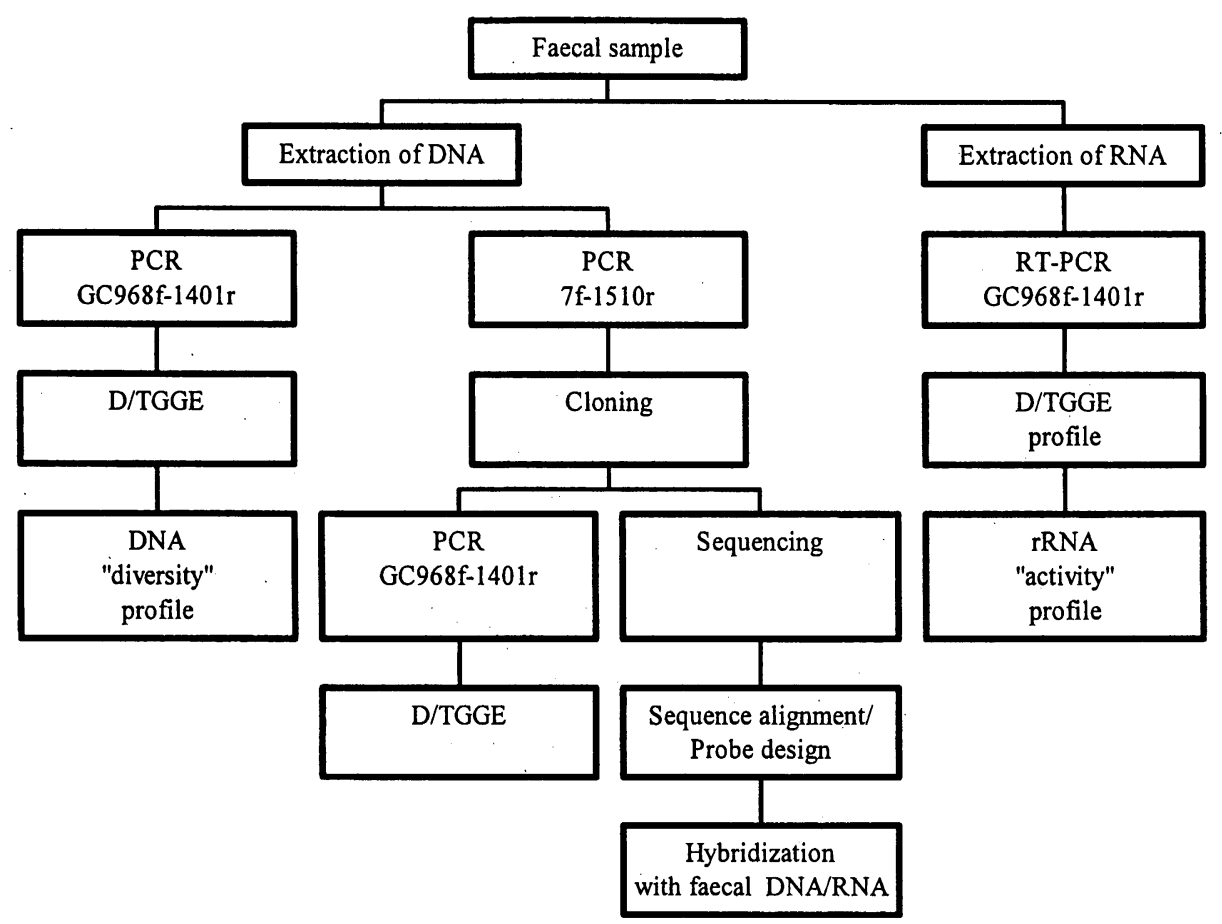

Fig. 1. Flow diagram of the DNA/RNA analysis.

trophoresis (D/TGGE). This approach was first applied in microbial studies by Muyzer and co-workers (18), leading to a cascade of applications in the field of microbial ecology. In most of these studies the DGGE fingerprints were made to give a qualitative view of the community. More recently, a quantitative analysis was made by estimating the intensities of the bands in the DGGE profile and by comparing those with internal standards. Competitive reverse transcriptase (RT)-PCR has been applied to quantify dominant bacterial groups in soil (8). The complexity of the profiles can also be expressed by estimating the Shannon diversity and equitability indices proposed by Shannon and Weaver (25), as implemented by Begon and co-workers (5). In a recent study on the effects of 3-chlorobenzoate on the composition of the microbiota in soil, Ramirez Saad demonstrated the value of Shannon indices to describe complex banding patterns (Refs. 19, 21).

In the current paper we will focus on the use of the DGGE and TGGE techniques in characterising bacterial communities in the GIT and faeces of humans and related animals. We briefly will describe how the human gut is colonised after birth and to what extent the composition is influenced by the genetics of the host and its diet.

\section{MATERIALS AND METHODS}

Faecal samples were collected from healthy volunteers and mammals from the zoo. Samples from mature volunteers were stored at $-20^{\circ} \mathrm{C}$ and subsequently processed as described previously (28). Faecal samples from babies were stored at $4{ }^{\circ} \mathrm{C}$ and processed within $12 \mathrm{hr}$ after collection (Favier, Akkermans, de Vos, unpubl.). The processing of the samples followed the general steps as indicated in Fig. 1. Major community shifts were described as changes in the DGGE profile of the 16S rDNA amplicons. The PCR and RT-PCR reactions targeting the $\mathrm{V} 6$ to $\mathrm{V} 8$ variable regions of respectively the $16 \mathrm{~S}$ rDNA or rRNA were set up according to Felske et al. (9). The amplicons obtained from faecal DNA and rRNA were separated on a TGGE system (Diagen, Düsseldorf, Germany) (8) or on a DGGE system (Dcode, BioRad). Similarity indices of the fingerprints were calculated with Molecular Analyst (version 1.12) software package (BioRad, Hercules, Cal., USA).

\section{RESULTS AND DISCUSSION}

During the past few years an increasing number of papers has been published on the application of $16 \mathrm{~S}$ rRNA sequence diversity in the environment. In our studies on microbial communities in faeces we follow 
the strategy as shown in Fig. 1. Amplicons of 16S rDNA derived from PCR with universal primer pairs (GC968f1401r) using environmental DNA as target, can rapidly be separated by DGGE or TGGE. The banding patterns in the gel represent fingerprints of the dominant bacterial community. Each band represents at least one sequence derived from (occasionally more than) one bacterial operational taxonomic unit (OTU). Analysis by DGGE or TGGE allows separation of sequences only differing by at least one to a few nucleotides on a stretch of about 400 nucleotides. The resulting T/DGGE banding patterns give a quantitative view of the composition of the bacterial community, providing there is no bias in the PCR and that all 16S rDNA sequences are amplified with equal efficiency. Current studies indicate that this assumption is true for the primer combination tested when careful controls are included (8). In practice several factors can interfere with DGGE analysis: the cell lysis is never $100 \%$, the copy number of the gene may vary among strains from 1-10, and preferential amplification during PCR might occur. These drawbacks are partly eliminated by using rRNA rather than rDNA as target $(8,9)$. "Activity" profiles (Fig. 1), derived from RT-PCR products of environmental 16S, rRNA slightly differ from those derived from rDNA as target and probably better reflect the active part of the community $(8,9,21,28,29)$. Certain bands in the D/TGGE profiles are more distinct than other bands. This may be due to differences in metabolic activity among bacterial strains $(7,8)$ since protein synthesis is strongly related to the ribosome content in fast-growing bacteria. In this approach we do not differentiate between many low active cells with low ribosome content or small numbers of highly active cells with high numbers of ribosomes. The current insight in microbial communities does not give yet an absolute quantitative view of the reality but certainly helps us to understand shifts in the community as affected by environmental changes. Interpretation of DGGE fingerprints by estimating the similarity index is a powerful way to quantify complex shifts in the community. This approach was used to quantify shifts in the GIT community in pigs after weaning (Wei Yun Zhu, Williams, Akkermans, unpubl.) and human babies (Favier, Akkermans, de Vos, unpubl.). We have tested this approach by comparing DGGE profiles of individuals with different genetic relation, environment and diet. We report here on three different factors that influence the composition of the GIT-microflora: a. composition of the diet, $b$. physiological condition of the host, and c. host genetics. So far, only the effect of environmental on microbial diversity has been analysed in faecal samples.

\section{a. Effect of the Diet}

At birth the GI-tract is free from bacteria and the newborn baby receives antibodies with the milk from the mother to protect invasion by pathogens. During and immediately after birth bacteria from the faeces and skin of the mother may enter the "milieu interieur" of the baby and some of these will colonise the intestine. Remarkable differences have been observed among the bacterial strains colonising different individuals. Although many different bacteria enter the GI-tract only few will colonise and most others do not find a proper niche or are immobilised by the antibodies obtained from the mother. Major changes in the community structure occur during the first period of weaning and early years of life as indicated by the gradual changes in the DGGE profiles in time (Favier, Akkermans, de Vos, unpubl.). The DGGE patterns as well as the clone libraries of $16 \mathrm{~S}$ rDNA sequences always showed Clostridium sequences as the first major bacterial signal in the GIT. This is in contrast with earlier results based on plate counting where facultative anaerobes were found as the first organisms present in the GIT (26). The reason for this discrepancy may be due to bias in the plate counting techniques, although preferential amplification in the PCR can not be excluded. Three days after birth Bifidobacterium strains colonise the GIT of breast-fed babies (16). After weaning the microbial community becomes increasingly complex and more bacterial groups are able to survive in the GItract. In a comparative study on mature individuals from Finland and The Netherlands, we have demonstrated that the composition of the bacterial microflora is individually different but quite constant in time $(28,29)$. Few bands in the profiles were present in all adults, living in different countries (Finland, Ireland, Japan, Mexico, The Netherlands). The bands were identified by screening and sequencing of cloned $16 \mathrm{~S}$ rDNA. At least three sequences showing the highest homology (\% in brackets) to Ruminococcus obeum (93.1\%), Eubacterium halii (98.7\%), and Fusobacterium prausnitzii $(91.5 \%)$ were present in all human subjects and are likely to play a universal role in the GI-tract.

Changes in diet may effect part of the microbiota. Several authors have demonstrated that addition of oligosaccharides and other prebiotics could increase the number of bifidobacteria. Toxic compounds, like bean lectins, that target the epithelial cells in the intestine, may induce diarrhoea due to rapid growth of patho- 
genic Escherichia coli (20). Apart of these pathogenic effects the GIT microbiota is remarkably stable and changes in diet will not influence the composition of the predominant bacterial groups of the microbiota (28).

\section{b. Physiological Condition of the Host}

Change in the health of the host is often correlated with major changes in the GIT microbiota in which opportunistic GIT-pathogens can rapidly dominate. Many studies have been published on this subject, but all have been based on traditional plate counts of bacteria. After recovery of an intestinal disorder, the GIT microbiota is restored in a stable complex community. Very little information is available on the real composition of such a stable "healthy microbiota." Is the composition of the GIT microbiota before and after e.g. diarrhoea really the same? To what extent affect antibiotics and other drugs the composition of the microbiota? It will be clear that further studies are needed to define what a "healthy microbiota" means and how this bacterial community responds to environmental changes in the GIT. Although this subject has been studied for several decades (see reviews Refs. 12, 27), the results have to be interpreted with caution, because of the bias in the traditional plate counting technique.

\section{c. Host Genetics}

Recent studies on the distribution of methane-production in faecal samples revealed unequal occurrence in the animal kingdom (13). In humans some individuals are known as methane-producers, while others fail to produce methane. By comparing the phylogenetic position of the animals with the occurrence of GIT methane production, Hackstein demonstrated that once the ability to produce methane was lost, this never appeared again in the evolutionary tree. To explain this phenomenon he proposed the existence of eukaryal genes that are involved in the recognition of methanogens. It is tempting to suggest that bacterial-host recognition is a general phenomenon as has been observed previously in plant-microbe symbiosis. In a comparative study on the composition of the faecal microflora from adults we observed that DGGE profiles of most individuals were remarkably constant in time (28). In an extended family study it appeared that faecal $16 \mathrm{~S}$ rDNA-DGGE profiles of genetically related persons were more similar than those of genetically unrelated persons in the same family (Zoetendal, et al., submitted). Figure 2 shows the DGGE profile of $16 \mathrm{~S}$ rDNA from 3 adult family members, viz. two heterozygous twins: brother (A), his sister (B) and wife of sub-

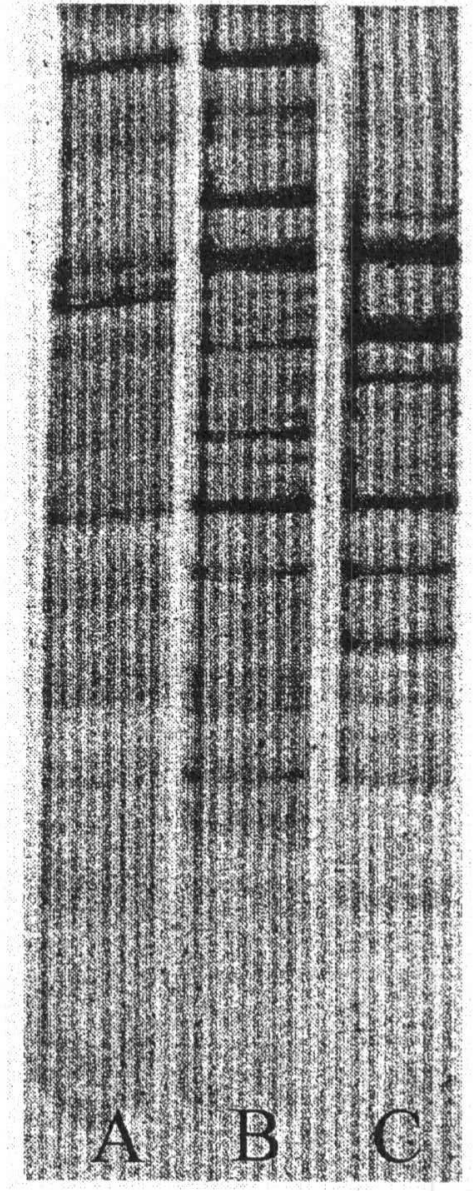

Fig. 2. DGGE profile of $16 \mathrm{~S}$ rDNA from 3 adult family members: male (A), his twin sister (B) and his wife (C).

ject $\mathrm{A}(\mathrm{C})$. The similarity index between brother (A) and sister (B) was 63.1 while the similarity index between husband (A) and wife (C) was only 41.5. Occasionally, profiles within a whole family were highly similar, indicating that the composition of an adult GIT microflora is determined by a complex of factors, including transmission of bacteria within a family, diet, and recognition by the host. This was further supported by DGGE profiles of mammals with a wider phylogenetic relationship, including cats, dogs, monkeys, camels and pigs: most of these profiles were genus-specific.

\section{CONCLUSIONS AND FUTURE PERSPECTIVES}

T/DGGE profiles of $16 \mathrm{~S}$ rDNA amplicons give a rapid view of the composition of the prominent members of a microbial community and can be used to detect changes within the community as a result of changes in the environmental conditions (diet, stress, probiotics, prebiotics). Despite of the power of this molecular approach we have to realise that the methods are PCR- 
based, and biases may occur. This means that T/DGGE fingerprints can not simply be converted to actual biomass. So far, most T/DGGE fingerprints were made with universal primers which only provides information about the predominant types. It is tempting to suggest that application of group-specific primers will give further insight in the occurrence of functionally relevant but less dominant bacteria. MPN-PCR, competitive PCR techniques and new primer sets are currently being developed and estimation of diversity indices, as is used in plant and animal ecology, is expected to contribute significantly to GIT-microbiology. Our knowledge of the behaviour of complex bacterial communities is still very preliminary and needs further studies in molecular microbial ecology of the GIT.

\section{REFERENCES}

(1) Akkermans ADL. 1998. Molecular tools for tracking micro-organisms in the environment. In Microbial Interactions in Agriculture and Forestry, Vol 1, Subba Rao NS, Dommergues Y (eds), Science Publishers, Enfield, NH, USA, p. 1-18.

(2) Akkermans ADL, Mirza MS, Harmsen HJM, Blok HJ, Herron PR, Sessitsch A, Akkermans WM. 1994. Molecular ecology of microbes: a review of promises, pitfalls and true progress. FEMS Microbiol Rev 15: 185-194.

(3) Akkermans ADL, Van Elsas JD, de Bruijn FJ. (eds). 1999. Molecular Microbial Ecology Manual, incl Supplements 1-4, Kluwer Academic Publishers, Dordrecht.

(4) Amann RI, Ludwig W, Schleifer KH. 1995. Phylogenetic identification and in situ detection of individual microbial cells without cultivation. Microbiol Rev 59: 143-169.

(5) Begon M, Harper JL, Townsend CR. 1960. Ecology: Individuals, Populations and Communities, Blackwell Scientific Publications, Oxford, UK.

(6) Colwell RR, Brayton PR, Grimes DJ, Roszak DR, Huq SA, Palmer LM. 1985. Viable but nonculturable Vibrio cholerae and related pathogens in the environment: implications for the release of genetically engineered microorganisms. Bio/ Technology 3: 817-820.

(7) Felske A. 1999. Response of predominant soil bacteria to grassland succession as monitored by ribosomal RNA analyses. PhD Thesis, Wageningen Agricultural University, The Netherlands, 113 pp.

(8) Felske A, Akkermans ADL, de Vos WM. 1998. Quantification of $16 \mathrm{~S}$ rRNAs in complex bacterial communities by multiple competitive RT-PCR in TGGE fingerprints. Appl Environ Microbiol 64: 4581-4587.

(9) Felske A, Engelen B, Nübel U, Backhaus H. 1996. Direct ribosome isolation from soil to extract bacterial rRNA for community analysis. Appl Environ Microbiol 62: 41624167.

(10) Finegold SM, Sutter VL, Mathisen GE. 1983. Normal indigenous intestinal flora. In Human Intestinal Microflora in Health and Disease, Hentges DJ (ed), Academic Press,
New York, p. 3-31.

(11) Fischer SG, Lerman LS. 1979. Length-independent separation of DNA restriction fragments in two-dimensional gel electrophoresis. Cell 16: 191-200.

(12) Gibson GR, Macfarlane GT. (eds). 1995. Human Colonic Bacteria: Role in Nutrition, Physiology and Pathology, CRC Press.

(13) Hackstein JHP, van Alen TA. 1996. Fecal methanogens and vertebrate evolution. Evolution 50: 559-572.

(14) Holdeman LV, Good IJ, Moore WEC. 1976. Human fecal flora: variation in bacterial composition within individuals and a possible effect of emotional stress. Appl Environ Microbiol 31: 359-375.

(15) Internet: http://www.cme.msu.edu/RDP/analysis.html

(16) Kok RG, de Waal A, Schut F, Welling GW, Weenk G, Hellingwerf KJ. 1996. Specific detection and analysis of a probiotic Bifidobacterium strain in infant feces. Appl Environ Microbiol 62: 3668-3672.

(17) Maidak BL, Olson GJ, Larsen N, Overbeek R, McCaughey MJ, Woese CJ. 1997. The RDP (Ribosomal Database Project). Nucleic Acids Res 25: 109-111.

(18) Muyzer G, de Waal EC, Uitterlinden GA. 1993. Profiling of complex populations by denaturing gradient gel electrophoresis of polymerase chain reaction-amplified genes coding for 16S rRNA. Appl Environ Microbiol 59: 695-700.

(19) Nübel U, Garcia-Pinchel F, Kühl M, Muyzer G. 1999. Quantifying microbial diversity: morphotypes, 16S rRNA genes, and carotenoids of oxygenic phototrophs in microbial mats. Appl Environ Microbiol 65: 422-430.

(20) Pusziai A, Grant G, Spencer RJ, Duguld TJ, Brown DS, Ewen SWB, Peumans WJ, van Damme EJM, Bardocz S. 1993. Kidney bean lectin-induced Escherichia coli overgrowth in the small intestine is blocked by GNA, a mannose-specific lectin. J Appl Bacteriol 75: 360-368.

(21) Ramirez Saad HC. 1999. Molecular Ecology of Frankia and other soil bacteria under natural and chlorobenzoatestressed conditions. PhD Thesis, Wageningen Agricultural University, The Netherlands, $119 \mathrm{pp}$.

(22) Rosenbaum V, Riesner D. 1987. Temperature gradient gel electrophoresis-thermodynamic analysis of nucleic acids and proteins in purified form and in cellular extracts. Biophys Chem 26: 235-246.

(23) Roszak DB, Grimes DJ, Colwell RR. 1984. Viable but nonrecoverable stage of Salmonella enteritidis in aquatic systems. Can J Microbiol 30: 334-338.

(24) Savage DC. 1977. Microbial ecology of the gastrointestinal tract. Annu Rev Microbiol 31: 107-133.

(25) Shannon CE, Weaver W. 1963. The Mathematical Theory of Communication, University of Illinois Press, Urbana.

(26) Stark PL, Lee A. 1982. The microbial ecology of the large bowel of breast-fed and formulae-fed infants during the first year of life. J Med Microbiol 15: 189.

(27) Tannock GW (ed). 1999. Probiotics: A Critical Review, Horizon Scientific Press, Wymondham, Norfolk, UK.

(28) Zoetendal EW, Akkermans ADL, de Vos WM. 1998. Temperature gradient gel electrophoresis analysis of $16 \mathrm{~S}$ rRNA from human fecal samples reveals stable and host-specific communities of active bacteria. Appl Environ Microbiol 
64: 3854-3859.

(29) Zoetendal EG, Akkermans AD, de Vos WM. 2000. Molecular characterization of microbial communities in the GI-tract based on $16 \mathrm{~S}$ rRNA sequence diversity. In New
Approaches for the Generation and Analysis of Microbial Fingerprints, Dijkshoorn L, Towner KJ, Struelens M (eds), Elsevier Science, Amsterdam. 